\title{
Novel Cancer Immunotherapies and Antitumor Immunity
}

\author{
Lei Zhao $\mathbb{D},{ }^{1}$ Jianjun Zhang, ${ }^{2}$ Liang $X u\left(\mathbb{D},{ }^{3}\right.$ and Hussein A. Abbas $\mathbb{D}^{2}$ \\ ${ }^{1}$ PLA General Hospital, Beijing, China \\ ${ }^{2}$ The University of Texas, MD Anderson Cancer Center, Houston, USA \\ ${ }^{3}$ Beijing Institute of Pharmacology and Toxicology, Beijing, China \\ Correspondence should be addressed to Lei Zhao; jackyzhao010@126.com
}

Received 14 April 2019; Accepted 12 June 2019; Published 22 July 2019

Copyright (C) 2019 Lei Zhao et al. This is an open access article distributed under the Creative Commons Attribution License, which permits unrestricted use, distribution, and reproduction in any medium, provided the original work is properly cited.

Recent success of immune-checkpoint blockade therapy in clinic has revealed that blockade of tumor-associated immunosuppression and initiation of tumor-specific immunity could be critical for the development of novel immunotherapies against cancer. Deep understanding of how to initiate and modulate tumor-specific immunity would be critical for the development of novel cancer immunotherapies.

In this issue, there are 3 review articles that cover key immunological tools: bispecific antibodies, natural killer (NK) cells, and neoantigens. S. Chen et al. reviewed the current understanding of bispecific antibodies and its chronological development to produce these antibodies, mechanism of action, application in cancer medicine, and the economic impact of these drugs. S. Matosevic reviewed the emergence of NK cells as an adoptive immune-cellular therapy and the technical background behind its engineering from viral and nonviral vectors. R.-Y. Pan et al. reviewed the quest to identify tumor neoantigens and how to leverage these findings in immunotherapy-based platforms in preclinical and clinical models.

In order to complement the aforementioned articles, this issue also included 6 research articles that span different aspects of cancer immunology and therapeutics. I. Poláková et al. demonstrated the utility of deep immune-profiling of lymphoid and myeloid components in the blood and tumor tissue of head and neck cancer patients. Y. Wu et al. identified HLA-A2-based epitopes that can elicit cytotoxic T lymphocyte-based responses. Two monoclonal antibodies targeting EGFR in animal studies were also included here. W. Qiu et al. discussed a novel monoclonal antibody tar- geting EGFR with better safety and efficacy profiles than cetuximab when combined with irinotecan in animal models. Y. Yang et al. demonstrated the efficacy of fusing Pseudomonas-based immunotoxin with a novel EGFRbased antibody in a murine model of esophageal cancer. Q. Zhang et al. demonstrated how combining the multikinase inhibitor, regorafenib, with CAR-NK cells could elicit responses in colorectal cancer cell lines.

Collectively, these articles integrate different aspects of immune-based therapies in the current management of cancer. Our current understanding of immune therapies and antitumoral immunity still requires significant refining. More studies are needed to identify the biomarkers of response to immunotherapies and CAR-based treatments so that nonresponders could be spared the risks of adverse events. Are there other checkpoint blockers that one could leverage in cancer treatment? Which patients would benefit from bispecific antibodies compared to CAR-based treatments? Is there a role for combination immune-based therapies with targeted therapy or chemotherapy? These are questions that are relevant in the decision process for cancer patients and require more in-depth and extensive research to address. Nevertheless, the current evidence strongly suggests that immune-based therapies work but understanding the context of its activity is vital for its success.

\section{Conflicts of Interest}

The guest editorial team gathered for developing the mentioned special issue comprising Assoc. Prof. Lei Zhao, 
Prof. Jianjun Zhang, and Assoc. Prof. Liang Xu provide a clear declaration that they do not have any conflict of interest or do not have any private agreements with companies.

\section{Acknowledgments}

We are very satisfied that our subject resulted in so many valuable papers, and we would like to thank all the authors who submitted their work for consideration to this special issue. Without their effort, this special issue would have not taken place. Editors would like to thank the reviewers who thoroughly revised the papers and provided important suggestions that significantly improved the papers.

Lei Zhao

Jianjun Zhang

Liang Xu

Hussein A. Abbas 


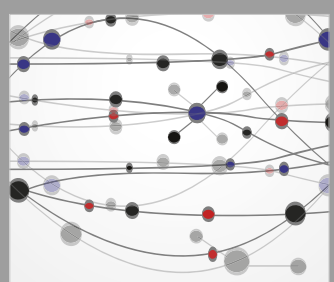

The Scientific World Journal
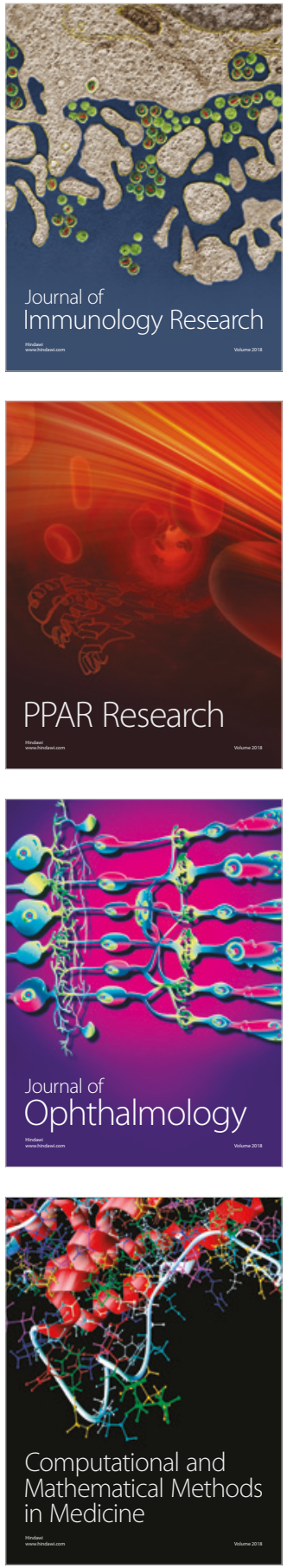

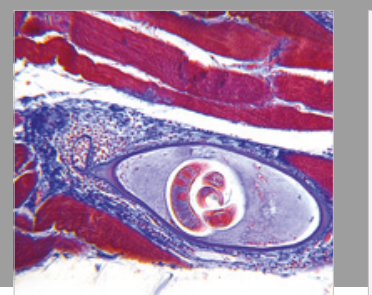

Gastroenterology Research and Practice

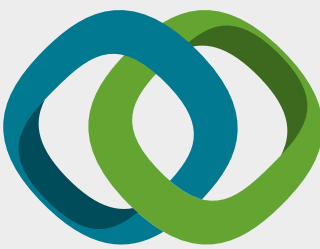

\section{Hindawi}

Submit your manuscripts at

www.hindawi.com
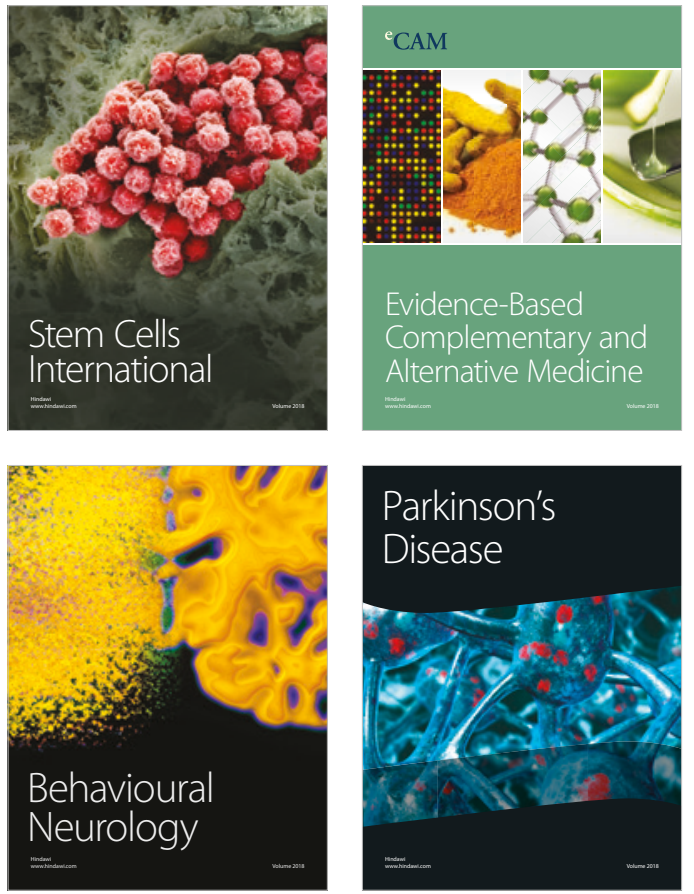

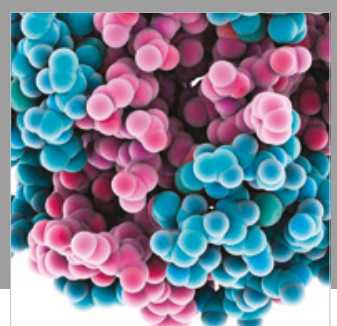

ournal of

Diabetes Research

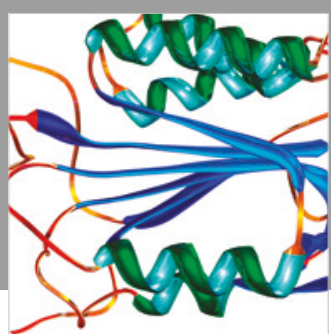

Disease Markers
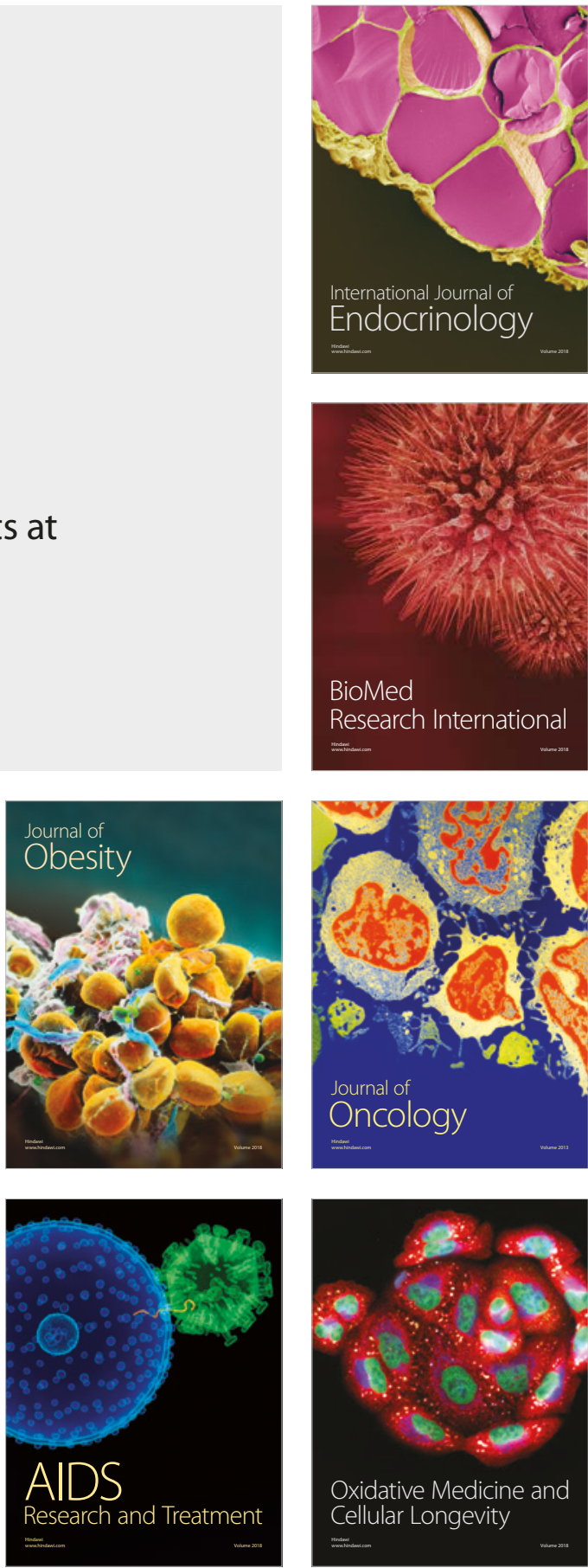